Original Research Paper

\title{
Infrared Thermography for the Characterization of Painted Vaults of Historic Masonry Buildings
}

\author{
Claudia Casapulla, Alessandra Maione and Luca Umberto Argiento \\ Department of Structures for Engineering and Architecture, University of Naples Federico II, Italy
}

\section{Article history}

Received: 26-01-2018

Revised: 06-02-2018

Accepted: 15-02-2018

Corresponding Author: Claudia Casapulla

Department of Structures for Engineering and Architecture, University of Naples Federico II, Italy

Email: casacla@unina.it

\begin{abstract}
The infrared thermography is a type of non-destructive investigation which can find interesting application in acquiring information about the structural features of ancient masonry buildings. In this case, in fact, the needs of preservation of the historical values have to be compound with the needs of achieving a proper level of knowledge of the parameters influencing the structural behaviour in order to perform a reliable evaluation of their seismic safety. A recent application of the infrared thermography was proposed in the framework of the Project on the seismic evaluation of the Museum of Capodimonte in Naples (Italy). The objective was to clarify if the painted vaults covering some rooms could have structural function and to identify their constructive typology. In this circumstance, an interesting methodological approach, with more general validity, has been developed by integrating the instrumental investigations with different sources of information, such as historical documents and hypotheses of critical interpretation.
\end{abstract}

Keywords: Masonry Vaults, Historical Buildings, Integrated Methodological Approach, Non-Destructive Testing (NDT) Methodologies

\section{Introduction}

The typological identification of the horizontal structures is a crucial aspect in defining proper models of the seismic behavior of masonry buildings. In fact, these structures influence the entity and the repartition of the seismic forces among the vertical walls by virtue of their property of mass and stiffness, while the effectiveness of their connection with the vertical structures ensures the occurrence of a global behavior preventing the out-of-plane mechanisms of the walls (Casapulla and Argiento, 2016; Casapulla et al., 2017a; Giresini and Sassu, 2017).

However the acquisition of the data required by a proper characterization of these structures is difficult to carry out, in particular when cultural heritage buildings are involved. In these cases, in fact, the execution of direct inspections and testing is usually not compatible with the needs to preserve the historical and artistic values inherent to painted ceilings or remarkable floors. Despite their limitations (Carlomagno et al., 2005), Nondestructive Testing (NDT) methodologies have lately gained increasing interest in this research field (Binda and Saisi, 2009; Grinzato et al., 2002), also thanks to the continuous improvement of technologies characterized by a low impact on the structures.
The attention of this paper is focused in particular on the infrared thermography, a NDT method which has been revealed effective for several kinds of investigations on historical buildings, because it does not require contact with the tested object and allows preserving its integrity (Avdelidis and Moropoulou, 2004; Meola, 2007; Spodek and Rosina, 2009). The infrared thermography is usually carried out according to two distinct approaches depending on the existing thermal contrast between the investigated object and the background: The passive approach detects the thermal radiation emitted by its surface under natural conditions, without employing any heating; this approach provides mainly qualitative data about the object. The active approach, instead, requires artificial heating usually obtained with the light emitted by flashed lamps, lasers or other light sources (Mercuri et al., 2011). This approach seems to be very promising in order to obtain also quantitative data thanks to the recent advances in thermographic signal processing. About the active approach, Maierhofer and Roellig (2009) presented several case studies showing the effectiveness of the impulse-thermography for the investigation of cracks, delaminations and masonry structure behind plaster. 
Both approaches, instead, were recently adopted by Cantini et al. (2013) in the study of the vault of the Assembly Room within the Palace of the Academy of Sciences in Torino; the active approach allowed detecting the presence of detached areas of the stuccowork, while the passive approach was used for the identification of the structural elements of the wooden frame of the vault.

An interesting application of the infrared thermography according to the passive approach was recently carried out by the authors of the present paper in the framework of the ARCUS-MiBACT Project on the "Assessment of the seismic safety of National Museums of Italy" (Prota et al., 2015) supported by the Ministry of the Cultural Heritage (MiBACT), in order to characterize the constructive typology of the vaults covering some rooms at the first level of the Capodimonte Museum in Naples. A multi-level approach proposed by the Italian Guidelines on Cultural Heritage was developed within this project (DCCM, 2011), as generally carried out to assess the seismic safety of masonry buildings (Casapulla et al., $2017 b$ ). In particular, the case study related to some painted vaults is described in this study, highlighting how the findings of the thermographic survey were critically interpreted and integrated by other source of information to achieve a proper characterization of the constructive features of these structures.

An Integrated Methodological Approach for the Identification of the Structural Features of Ancient Masonry Buildings

An interesting methodological approach was developed in the framework of the structural analysis of the Capodimonte Museum by integrating the instrumental investigations with different sources of information, such as historical documents and hypotheses of critical interpretation. Hence, a basic level of knowledge was firstly achieved through the visual inspections and the architectural survey; these activities together with the verification of the data derived from the documentary and bibliographic sources allowed identifying the essential geometrical and constructive features of the building. However, at the end of this first phase of knowledge some problematic aspects arose, requiring deeper investigations. They concern mainly the two following questions:

- The identification of the typologies of masonry and the attribution of these ones to the distinct phases of the construction history of the building.

- The characterization of the constructive typology of some vaults at the first level.

On the solution of the latter question, better described in the following section, is indeed focused the present paper.
Hence, in order to acquire the lacking data, a diagnostic strategy was defined with the objective of finding a compromise between the needs of preserving the historical values and the public function of the building on one side and the needs of modeling the structural behavior on the basis of a satisfactory knowledge of the constructive details, on the other side. To this aim, nondestructive techniques were chosen such as sonic tests for the vertical structures and infrared thermography for the analysis of the horizontal structures.

The diagnostic campaign was performed by Stressscarl district, Consorzio Tre and the Testing Laboratory at the Dist Department of the University of Naples.

\section{The Case Study of Some Masonry Vaults of the Capodimonte Museum in Naples}

The infrared thermography was used to characterize the constructive typology of the vaults related to the rooms labeled in Fig. 1 with their identification number and located at the first level of the Capodimonte Museum. The case study reported in this study is referred in particular to the three couples of adjacent rooms which are characterized by a similar configuration; these are indicated with the numbers 9 and 49, 10 and 48, 11 and 47 in Fig. 1. The vaults of these rooms are a type rather common in Italy known as a schifo or mirror vault and two of them (n. 9 and 10) are painted vaults (Fig. 2); all of them are supported by structural masonry walls on three sides, while on the fourth side there is only a thin wall of about $20 \mathrm{~cm}$ which divides the adjacent rooms. Hence, in order to understand whether these vaults have decorative or structural function the following hypotheses were formulated on the basis of the critical analysis of the historical documentation and of the geometrical relief.

According to a first hypothesis, the two adjacent rooms derive from the partition of a unique space covered by a structural masonry vault, maybe required by the exhibition needs of the Museum. With this transformation, three sides of the original vault were preserved, while a wooden structure was built on the fourth one, resembling the profile of the vault. This hypothesis is supported by the inclination of the fourth side of the vault that appears considerably more pronounced.

Another possible hypothesis is that the present visible vaults are not load-bearing, but they have a timber structure that repeats the shape of the original masonry vault. This kind of solution, on the other hand, was found out in the rooms 51 and 52 (Fig. 3) which also result from the division of a unique space; in fact, by approaching the narrow space behind the room 52 (hosting the precious "Salottino di porcellana") it was possible to discover that under the original masonry vault, still in place, there was a timber structure consisting in a series of ribs completed with a mat of plaited canes and bricks on a sheet. The height at the keystone of this latter vault is lower than that of the original one. 


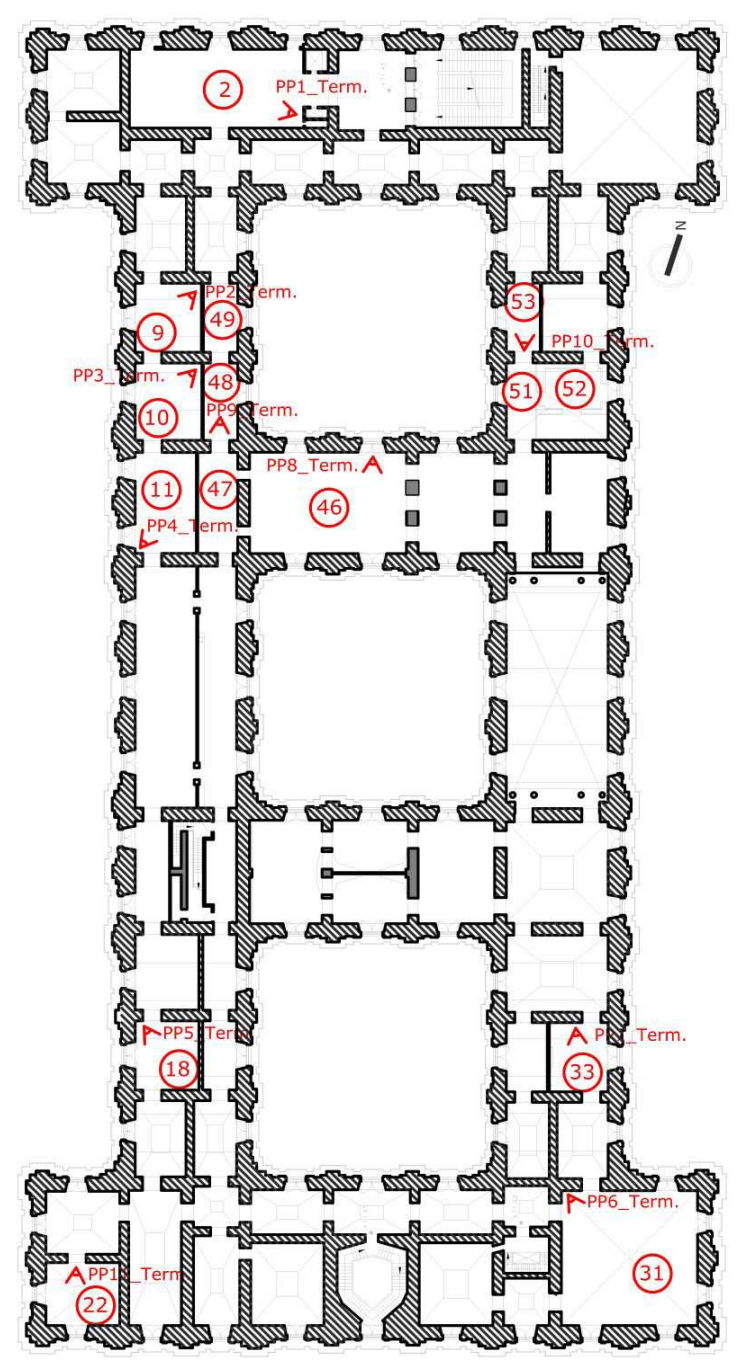

Fig. 1: Planimetric identification of the point of views of the infrared images together with the numbering of the rooms at the first level of the Capodimonte Museum

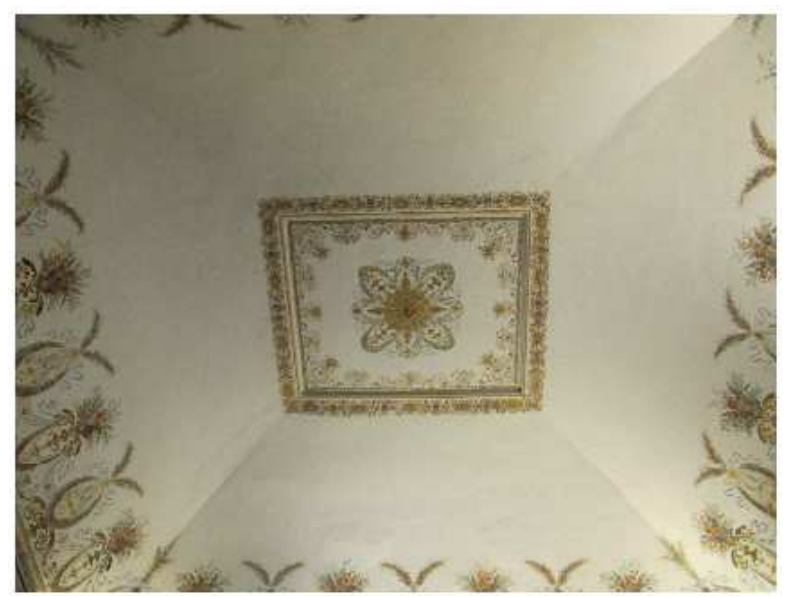

Fig. 2: Mirror painted vault of room n. 9

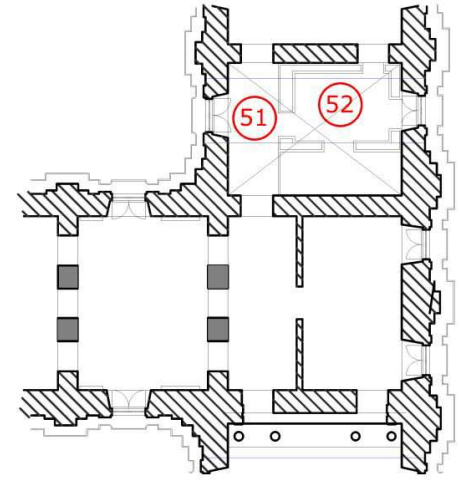

(a)

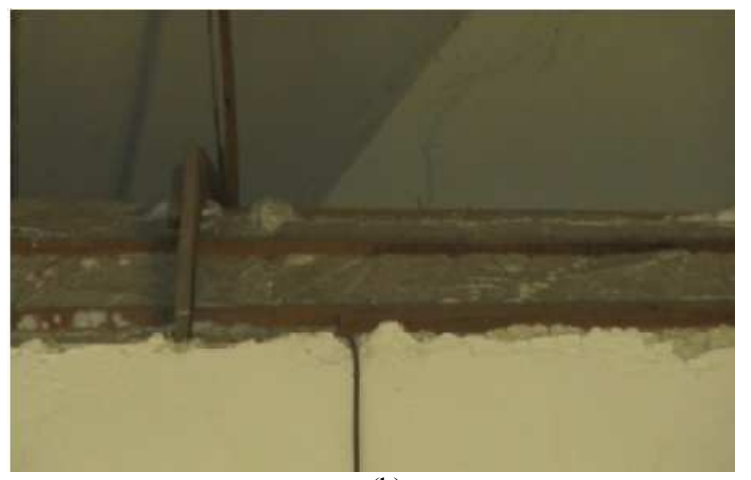

(b)

Fig. 3: (a) planimetric position of the "Salottino di Porcellana" (room n. 52); (b) timber vault visible from the room behind the "Salottino di Porcellana" under the original masonry vault

Hence it was supposed that a similar solution had been adopted for the rooms under study. In this case, the problem was also to understand whether the original vaulted structure was still in place or was replaced by a concrete slab.

Lastly, a third hypothesis assumes that the present visible vaults are structural masonry vaults but their fourth side, corresponding to the thin wall, is carried out by a concrete curb.

\section{The use of the Infrared Thermography Basic Theoretical Principles}

The thermographic surveys were carried out using an infrared camera (thermal camera) that allows detecting infrared energy emitted from an object, converting it into apparent temperature and displaying the result as an infrared image. In fact, all elements with a temperature above absolute zero emit heat and therefore radiation in the infrared field.

The thermal or infrared energy is characterized by a wavelength too large to be visible; it is, in fact, the portion of the electromagnetic spectrum that is perceived as heat. Thereafter the infrared cameras operate in wavelengths as long as $14000 \mathrm{~nm}(14 \mu \mathrm{m})$ while 
common cameras, forming images using visible light, operate in the range of 400-700 nanometers.

The existence of the infrared radiation was discovered in the $19^{\text {th }}$ century by William Herschel. He used a prism to produce a spectrum of colours and noticed that the temperatures of the colours increased from the violet to the red part of the spectrum. He also found that the highest temperatures are beyond the red portion of the spectrum in a region where no sunlight is visible.

The functional principle of the infrared thermography is governed by three essential physical laws:

- Kirchhoff's law defines the relation between emission and absorption of energy, which states that the absorption of a body has to equal its emissivity at every wavelength.

- Planck's law of radiation describes the distribution of the amplitudes of the energy that a black-body emits as radiation vs. different frequencies or wavelengths (i.e., spectral radiance).

- Stefan-Boltzmann law is applied to the emission of a surface over all wavelengths and states that the radiant power, I, grows with the fourth power of its absolute temperature.

The results of the infrared thermography are influenced by three essential factors: The surface configuration, the surface roughness and the environmental conditions.

The surface configuration influences the thermal conductivity that can be reduced if air voids and low density areas are present.

The surface roughness has the effect of increasing the surface emissivity with respect to that related to a smooth surface. This parameter is strictly related to the material characterizing the surface.

The environmental conditions such as solar exposition, temperature, wind speed, surface moisture that surrounds the surface affects the validity of the image interpretation.

\section{The Application of the Infrared Thermography to the Case Study}

For the thermographic investigations in the Capodimonte Museum the ThermaCAM B640 model (Fig. 4) of the FLIR Company was used. The system is provided of a Focal Plane Array detector operating in the band 7.5-13 $\mathrm{lm}$ and detects a temperature range from $40^{\circ} \mathrm{C}$ to $+120^{\circ} \mathrm{C}$ with a measurement accuracy of $\pm 2^{\circ} \mathrm{C}$. A passive approach of investigation was adopted by using the natural heat sources of the building as the solar radiation or the slow microclimate temperature.

In order to test the sensitivity of the camera, a preliminary thermographic scan of the vault covering the room n. 2 (Fig. 1) was carried out; here the presence of a timber structure (Fig. 5a and 5b) was considered very likely because the arrangement of this area together with the Auditorium at the ground floor dates back to $1950 \mathrm{~s}$; in that period the pre-existing Royal Chapel, that developed with its height along two levels, was completely transformed with the creation of an intermediate floor in order to ensure the continuity of the expositive path. Then this supposition was confirmed by the thermography as shown in Fig. 5b.

Figure 6-8 report the results of the thermographic survey related to the painted vaults covering the rooms $n$. 9 and 10 and to the vault of the room n. 11, respectively.

In particular, the thermographic image related to the latter vault (Fig. 8c) confirmed that it is not structural, as already suggested by the presence of a thin wall in common with the adjacent room n. 47.

On the other hand, also the vault covering this room has a timber structure, as revealed by the thermographic image of the similar room n. 48 in Fig. 9c.

It is very likely, therefore, that the timber structures were created after the partition of the unique space covered by the original vault. About the rooms n. 9 and 10, instead, their thermographic images do not reveal the signs of the timber skeleton and this may led to suppose the presence of masonry vaults. This result, however, seems to be not consistent with the presence of a nonstructural wall on one side, as previously highlighted. Then a plausible explanation of this configuration was derived from a further typology of vault (Fig. 10) that it was possible to see from a crawl space of the Farnesian staircase. It is a no-load-bearing masonry vault, characterized by a thin thickness of bricks disposed on a sheet $(20 \mathrm{~cm}$ average thickness). This typology could be compatible with the underneath thin wall and maybe it was related to the need of preserving the paintings on their intrados surface.

Lastly, the infrared thermography was also used to investigate the vaults related to the rooms $17,18,33$ e 46 (Fig. 1) and also in these cases it was possible to recognize from the infrared images of the intrados surfaces the presence of the ribs of a timber structure.

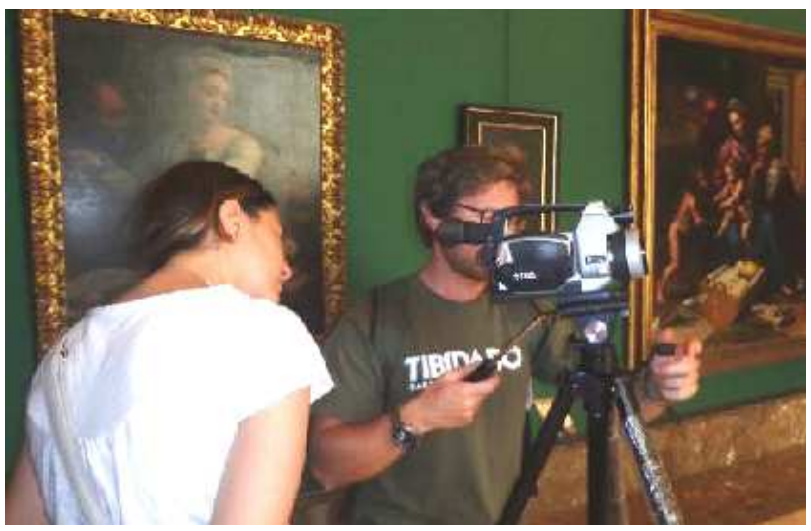

Fig. 4: The FLIR ThermaCAM B640 (infrared camera) 
Claudia Casapulla et al. / International Journal of Structural Glass and Advanced Materials Research 2018, Volume 2: 46.54 DOI: 10.3844/sgamrsp.2018.46.54

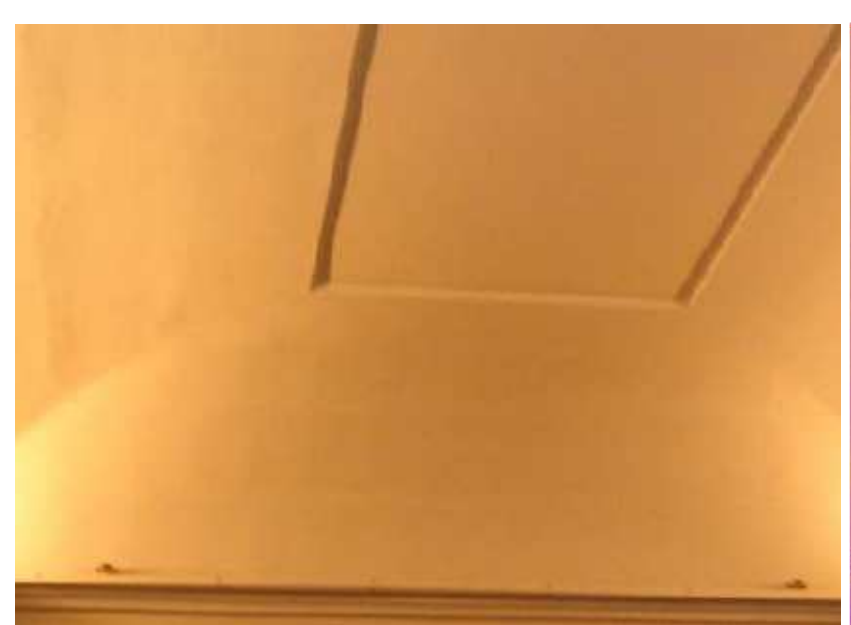

(a)

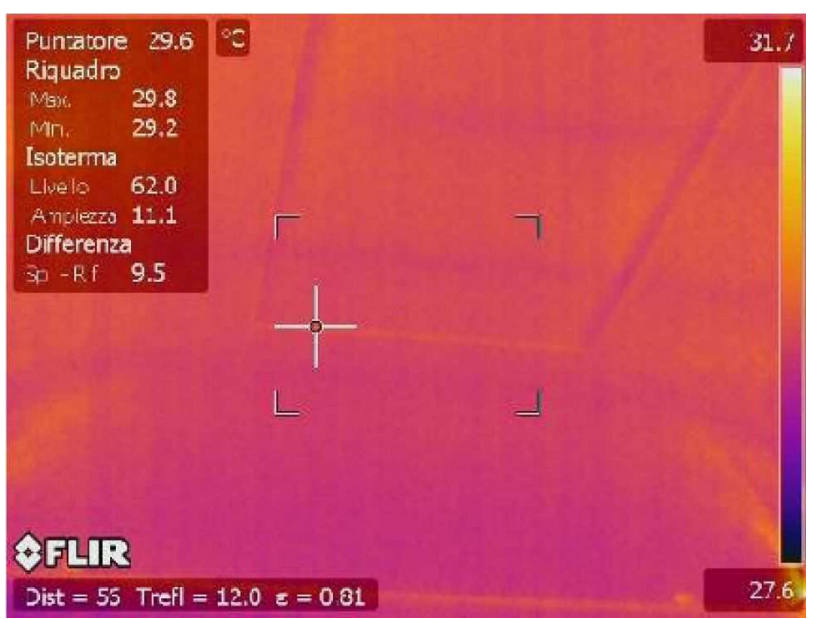

(b)

Fig. 5: Room n. 2. (a) view of the intrados of the vault (b) thermographic image showing the timber structure

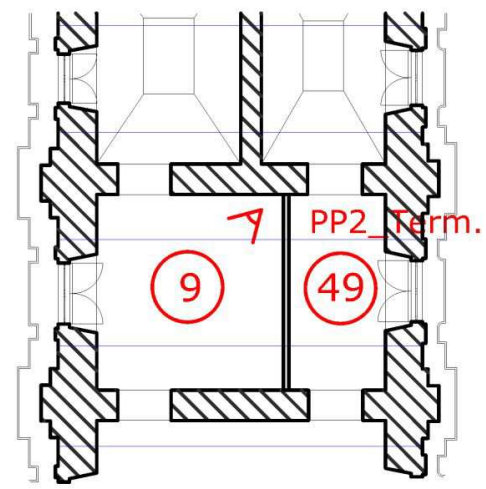

(a)

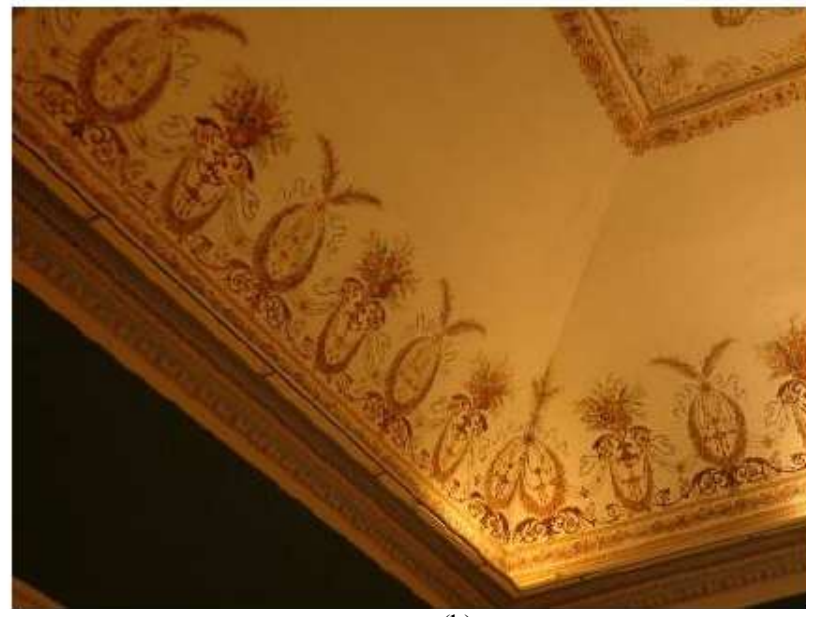

(b)

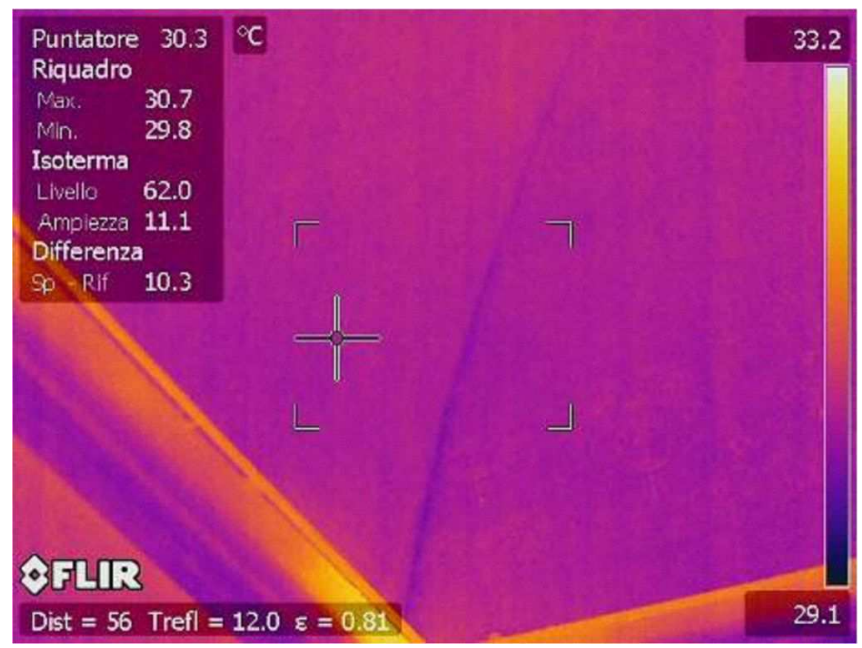

(c)

Fig. 6: Room n. 9. (a) planimetric localization of the point of view of the camera (b) view of the intrados of the vault (c) thermographic image 


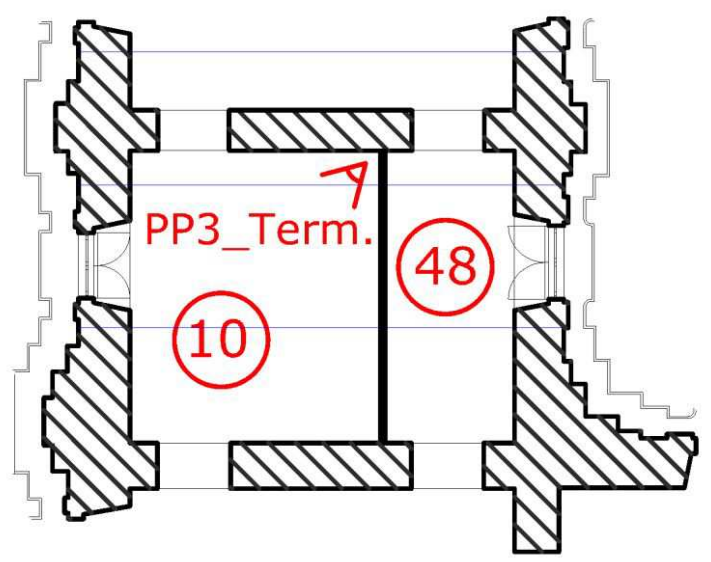

(a)

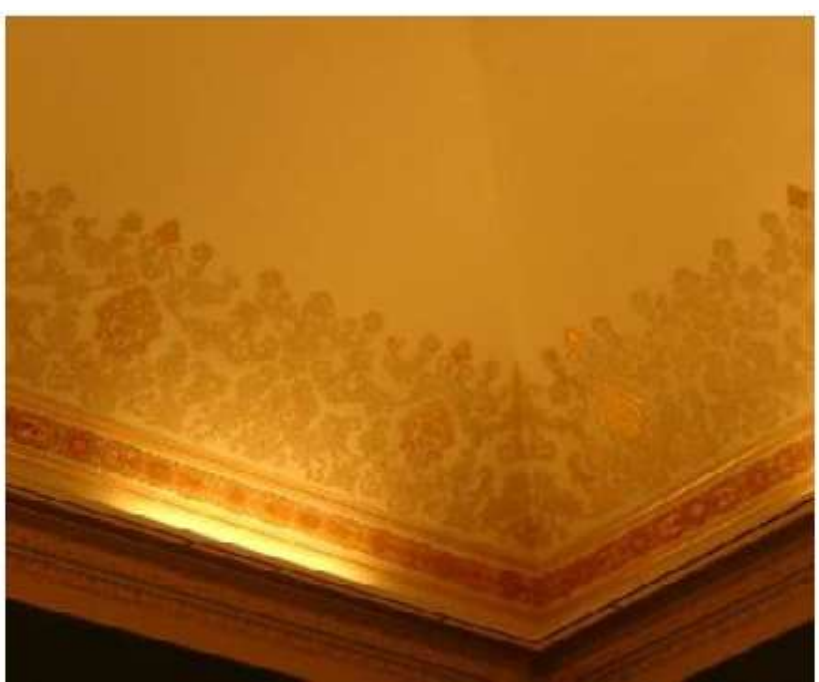

(b)

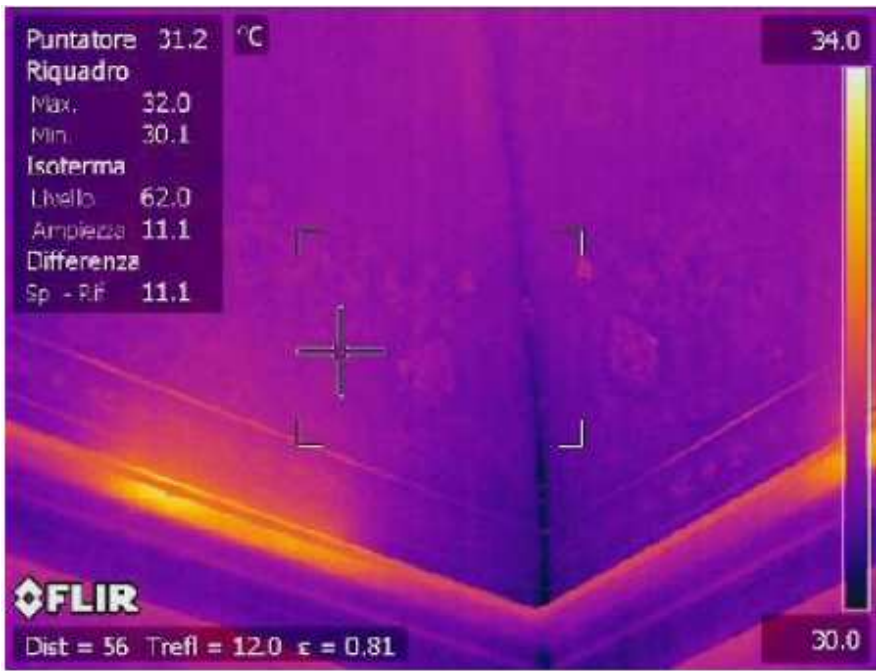

(c)

Fig. 7: Room n. 10. (a) planimetric localization of the point of view of the camera (b) view of the intrados of the vault (c) thermographic image

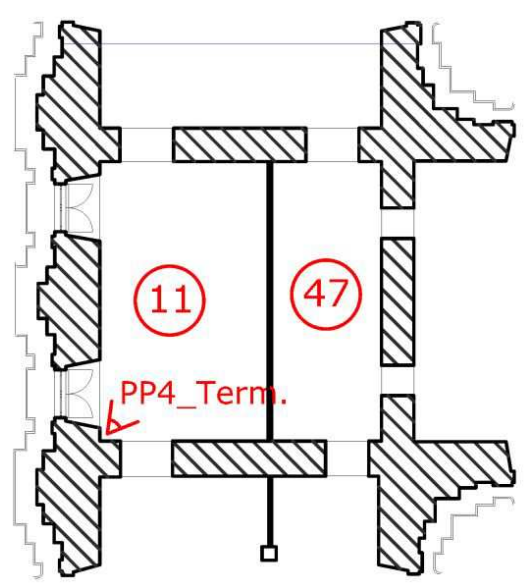

(a)

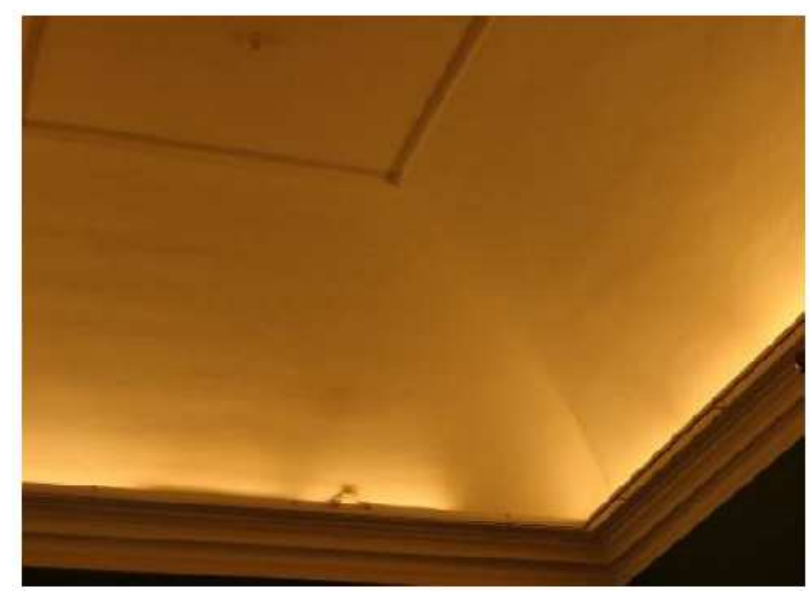

(b) 


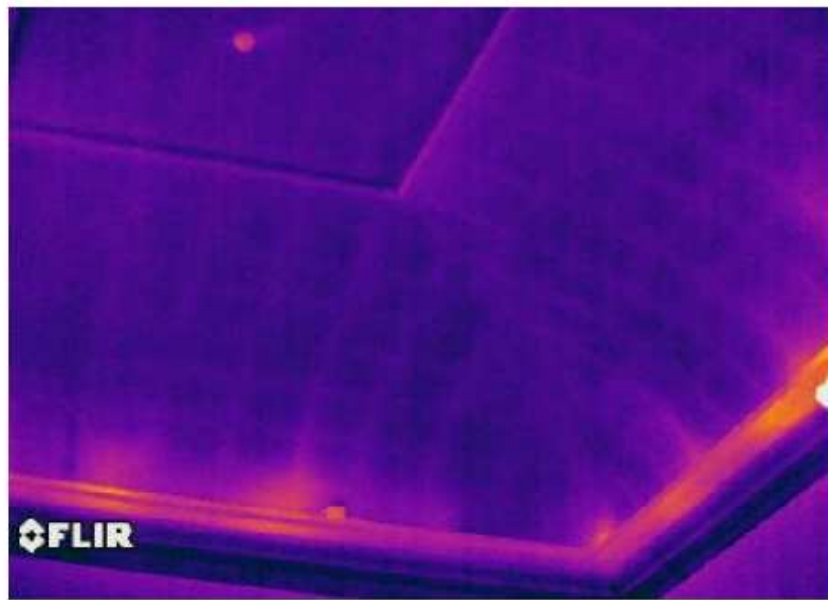

(c)

Fig. 8: Room n. 11. (a) planimetric localization of the point of view of the camera (b) view of the intrados of the vault (c) thermographic image

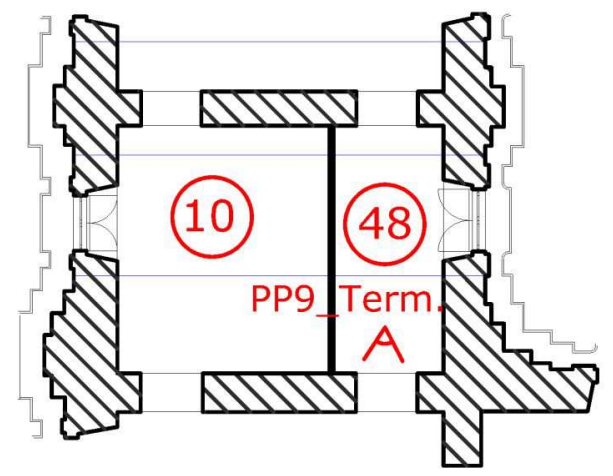

(a)

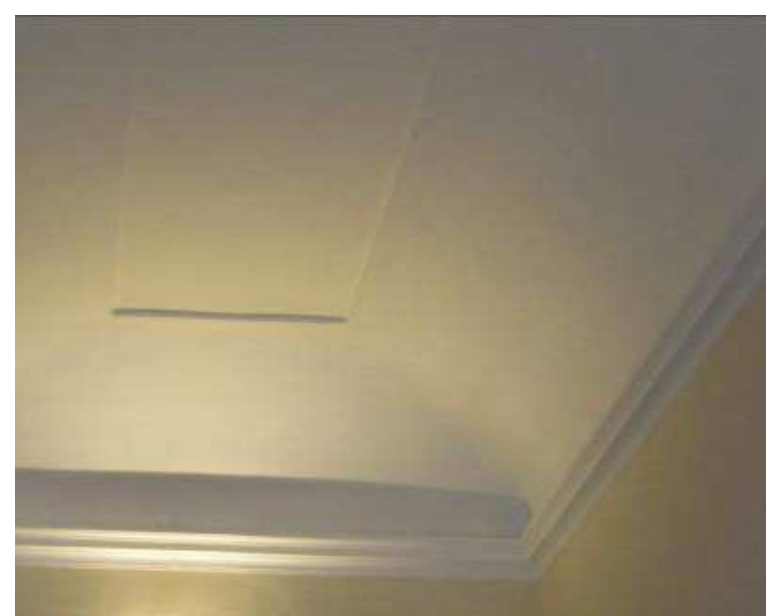

(b)

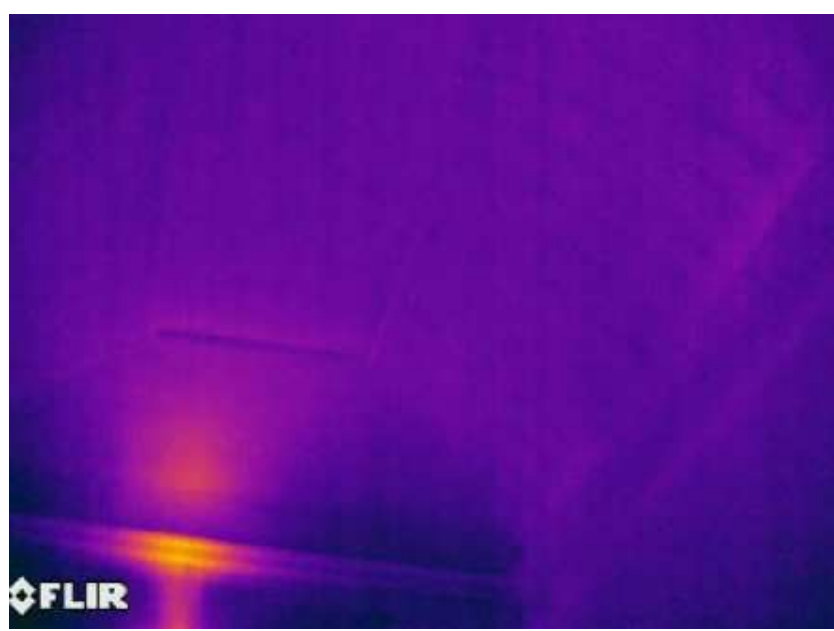

(c)

Fig. 9: Room n. 48. (a) planimetric localization of the point of view of the camera (b) view of the intrados of the vault (c) thermographic image 


\section{Discussion}

The critical interpretation of the instrumental findings in the light of the other information derived from the visual inspections and the analysis of the documentary sources has provided a complete characterization of the horizontal structures of the first level of the Capodimonte Museum. In particular, the infrared thermography has allowed a first distinction between structural masonry vaults and no- load-bearing vaults.

The first ones must withstand their self weight, the non-permanent structural loading and the variable loading; they have a prevailing configuration as a schifo or mirror vault and maximum height of about $8.8 \mathrm{~m}$; moreover they are dated back to the construction of the building and characterize the rooms of its north and south sides.

The no-load-bearing vaults can be referred to two typologies such as timber and masonry vaults; they must withstand only their self-weight, while the other loading are transferred to distinct and separated horizontal structures. The possibility to adopt the infrared thermography also from extrados could help to better identify possible structural vulnerabilities, completing the information from intrados of the vaults. However, the application of the passive infrared thermography form extrados was not suitable in this case because of the deep thickness of the floor slabs. Therefore, a proper classification of the no-load-bearing vaults on the basis of the typology of the overlying horizontal structures has to account for different sources of information; in some cases the visual inspections allowed to observe the structure of the timber vaults also from the extrados because they were built under the pre-existing masonry vaults that kept their load-bearing function. This is the already cited case of the "Salottino di porcellana", but also the case of the Library adjacent the hexagonal staircase (Fig. 11). The interest of these two cases is also related to the fact that they provide a good understanding of the constructive typology of this kind of vaults.

In other cases, where the overlaying load-bearing structure is not visible, the presence of structural masonry vaults was supposed on the basis of the finding of the anchors of ties, visible on the outer walls of the rooms. This is the case, indeed, of the rooms n. 9 and 10 described in the previous section.

Lastly, a further typology of no-load-bearing timber vaults is characterized by the presence of an overlaying plane diaphragm in r.c.; this solution is documented in particular for the rooms located in the transversal bodies around the central courtyard and dated back to the strengthening interventions carried out in the 1950s. Thus, it was supposed that a similar solution was adopted for the timber vault of the room n. 11 .

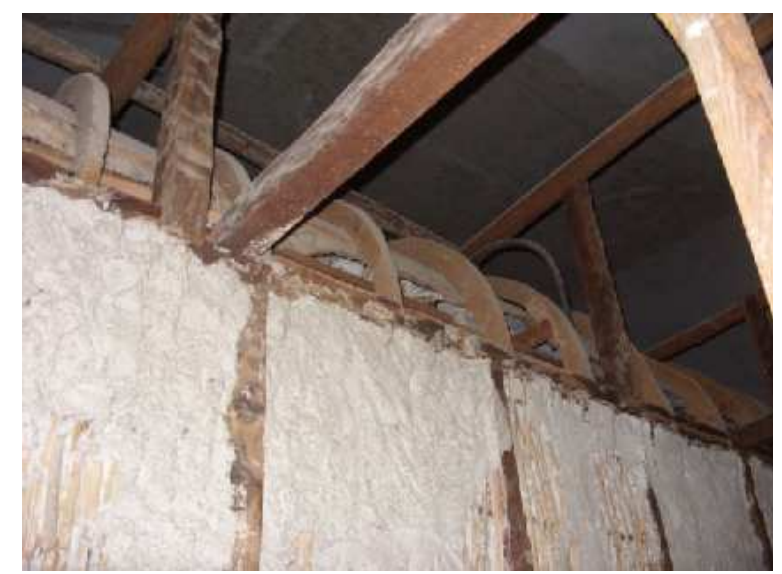

Fig. 11: Timber structure of the vault related to the Library

\section{Conclusion}

A case study related to the application of the infrared thermography to characterize the constructive typology of some vaults of the Capodimonte Museum in Naples was presented. This investigation campaign was carried out by the authors in the framework of the ARCUSMiBACT Project on the "Assessment of the seismic safety of National Museums of Italy" supported by the Ministry of the Cultural Heritage (MiBACT). The thermographic images of the intrados surfaces of the analyzed structures allowed a first distinction between structural masonry vaults and no-load-bearing vaults. For the latter category, two constructive typologies were recognized, as timber and masonry vaults. They are characterized by the presence of overlying horizontal structures, which have to fulfill the bearing function. In order to proper identify the features of these horizontal structures instrumental investigations have been critically interpreted and integrated by other information derived from the visual inspections and the critical analysis of the documentary sources.

This interesting methodological approach allowed achieving a complete characterization of the horizontal structures of the first level of the Capodimonte Museum and represented a basic requirement to define a reliable model of the structural behavior of the building aimed at the evaluation of its seismic safety.

\section{Acknowledgement}

This paper was presented at the 6th International Workshop on Design in Civil and Environmental Engineering, held at the University of Cagliari during November 9 - 10 - 11, 2017.

The authors acknowledge the sponsorship of Italian MiBACT through the ARCUS Project on the "Assessment of the seismic safety of National Museums of Italy" (2014-2015). We also wish to thank the Stress- 
scarl district that performed the diagnostic campaign. The project was coordinated by Prof. G. Manfredi and A. Prota, whose support is gratefully acknowledged.

\section{Author's Contributions}

Claudia Casapulla: She made contributions to conception and design and analysis and interpretation of data. She also coordinated the data-analysis and designed the structure of the text.

Alessandra Maione: She participated in all experiments, conceived the field of investigation and contributed to the writing of the manuscript.

Luca Umberto Argiento: He participated in all experiments, designed the research plan, organized the study and contributed to interpretation of data.

\section{Ethics}

This article is original and contains unpublished material. The corresponding author confirms that all of the other authors have read and approved the manuscript and there are no ethical issues involved.

\section{References}

Avdelidis, N.P. and A. Moropoulou, 2004. Applications of infrared thermography for the investigation of historic structures. J. Cultural Heritage, 5: 119-27. DOI: 10.1016/j.culher.2003.07.002

Binda, L. and A. Saisi, 2009. Application of NDTs to the diagnosis of Historic Structures. Proceedings of 7 th International Symposium on Non-Destructive Testing in Civil Engineering, (TCaE' 09), Nantes, France, pp: 1-27.

Cantini, L., C. Tedeschi, C. Tiraboschi and L. Binda, 2013. Use of Thermovision for the Survey of a Timber Vault in Torino. In: Nondestructive Testing of Materials and Structures, Güneş O., Y. Akkaya, (Eds.), Springer Science and Business Media, RILEM, ISBN-10: 9400707231, pp. 1203-1208.

Carlomagno, G.M., R. Maio, C. Meola and N. Roberti, 2005. Infrared thermography and geophysical techniques in cultural heritage conservation. Quantitative InfraRed Thermography J., 2: 5-24. DOI: 10.3166/qirt.2.5-24

Casapulla, C. and L.U. Argiento, 2016. The comparative role of friction in local out-of-plane mechanisms of masonry buildings. Pushover analysis and experimental investigation. Eng. Struct., 126: 158-173. DOI: 10.1016/j.engstruct.2016.07.036
Casapulla, C., L. Giresini and P.B. Lourenço, 2017a. Rocking and kinematic approaches for rigid block analysis of masonry walls: State of the art and recent developments. Buildings, 7: 69-69. DOI: $10.3390 /$ buildings 7030069

Casapulla, C., A. Maione and L.U. Argiento, 2017b. Seismic analysis of an existing masonry building according to the multi-level approach of the Italian guidelines on Cultural Heritage. Ingegneria Sismica, 34: 40-60.

DCCM, 2011. Directive of the chairman of the council of ministers, seismic risk evaluation and reduction of the cultural heritage. Proceedings of the Technical Rules for Constructions, (TRC' 11), Official Gazette of the Italian Republic.

Giresini, L. and M. Sassu, 2017. Horizontally restrained rocking blocks: Evaluation of the role of boundary conditions with static and dynamic approaches. Bull. Earthquake Eng., 15: 385-410. DOI: $10.1007 / \mathrm{s} 10518-016-9967-7$

Grinzato, E., P.G. Bison and S. Marinetti, 2002. Monitoring of ancient buildings by the thermal method. J. Cultural Heritage, 3: 21-9. DOI: 10.1016/S1296-2074(02)01159-7

Maierhofer, C. and M. Roellig, 2009. Active thermography for the characterization of surfaces and interfaces of historic masonry structures. Proceedings of the 7th International Symposium on NonDestructive Testing in Civil Engineering, (TCE' 09), Nantes, France, pp: 1-6.

Meola, C., 2007. Infrared thermography of masonry structures. Infrared Phys. Technol., 49: 228-233.

Mercuri, F., U. Zammit, N. Orazi, S. Paoloni and M. Marinelli et al., 2011. Active infrared thermography applied to the investigation of art and historic artefacts. J. Thermal Anal. Calorimetry, 104: 475-85. DOI: $10.1007 / \mathrm{s} 10973-011-1450-8$

Prota, A., G. Manfredi, C. Casapulla, L.U. Argiento and R. Frascadore et al., 2015. Museo di Capodimonte. Report of the ARCUS-MIBACT project on the "Assessment of the seismic safety of National Museums of Italy. Application of O.P.C.M. 3274/2003 S.M.I. and of the Directive P.C.M. 12.10.2007.

Spodek, J. and E. Rosina, 2009. Application of infrared thermography to historic building investigation. J. Architectural Conservat., 15: 65-81. DOI: 10.1080/13556207.2009.10785040 